\title{
Hepatic Connective Tissue Changes in Hepatosplenic Schistosomiasis
}

\author{
ZILTON A. ANDRADE, MD, EDIRIOMAR PEIXOTO, MD, \\ SYLVIANE GUERRET, BS, AND JEAN-ALEXIS GRIMAUD, MD
}

\begin{abstract}
Destruction of intrahepatic portal vein branches with dispersion of smooth muscle cells into the periportal fibrosis and preservation of arterial and ductal structures were the main characteristic findings seen in 66 surgical liver biopsies from patients with the hepatosplenic form of schistosomiasis. Besides these diagnostic features, the present histologic, immunocytochemical, and ultrastructural study revealed the presence of a complex matrix forming the portal and septal fibrosis in advanced schistosomiasis. There was marked hyperplasia of elastic tissue, presence of several collagen isotypes (I, III, procollagen III, IV, and V), actin, desmin, fibronectin, and laminin in a richly vascularized connective tissue. Signs of multifocal matrix (collagen) degradation were observed both at light and electron microscopic levels, suggesting a predominance of a fibrolytic process, at the time parasite-related lesions had almost disappeared. The latter findings are related to the involution of periportal fibrosis now being observed in patients who have undergone antischistosomal chemotherapy. They exemplify morphologic changes connected with chronic collagen degradation in human schistosomiasis that are similar to those first seen in experimental material. Evidence of either persistent or active chronic hepatitis was seen in several cases but its etiology could not be determined. Hum PATHOL 23: 566-573. Copyright (C) 1992 by W.B. Saunders Company
\end{abstract}

Schistosomiasis is one of the most prevalent parasitic diseases in the world. The World Health Organization estimates that more than 200 million people are infected and that 500 to 600 million are exposed to infection.' Five schistosome species can infect man but only one, Schistosoma mansoni, is found in the New World, especially in Brazil. The paired worms in this species live inside the veins of the mesenteric system and lay eggs in the intestinal mucosa. Eggs are frequently carried into the liver by the blood flow and are trapped in the portal radicles, inducing granulomas.

Advanced hepatic schistosomiasis is clinically represented by the hepatosplenic form of the disease, with periportal fibrosis, intrahepatic portal vein obstruction, and portal hypertension. Its incidence is less than 5\% of all infected people in endemic zones of Brazil. Its pathogenesis is related to high worm burden, ${ }^{2}$ coupled with a host inability to immune-modulate egg-induced lesions. ${ }^{3,4}$ Gross hepatic pathology is highly characteristic

From the Department of Pathology, Comçalo Moniz. Research Center, Bahia, Brazil; the Department of Surgery, Roberto Santos Central Hospital, Bahia, Brazil; and the Service of Cellular Pathology of the Liver, Institut Pasteur, I von, France. Accepted for publication July $11,1991$.

Key words: periportal fibrosis, pipe-stem fibrosis, schistosomiasis, hepatosplenic disease, collagen degradation.

Supported by FINEP (Brazil) and the CNPq/INSERM Cooperative Program

Address correspondence and reprint requests to Zilton A. Andrade, MD, Rua Valdemar Falcão, 121 (Brotas) 4l.945 Salvador, Bahia Brazil.

Copyright 61992 by W.B. Saunders Company

$(0046-8177 / 92 / 2905-0015 \$ 5.00 / 0)$ and sinec 1904 has becn known as "clay pipestem fibrosis". The histopathology of hepatic schistosomiasis in its advanced form also has been well documented in the literature. ${ }^{6-x}$ However, the application of different morphologic techniques to abundant material from human biopsies has revealed new findings that justify the present report. These new aspects are related to the potential for reversibility of the portal changes, to the participation of elastic tissue in schistosomal fibrosis, to associated changes of chronic hepatitis, to the peculiar behavior of the portal vein smooth muscle fibers, and to the dynamics of matrix changes in schistosomal portal and septal fibrosis.

\section{MATERIALS AND METHODS}

\section{General Clinical Data}

A total of 66 surgical biopsies of the liver were obtained from hepatosplenic patients undergoing splenectomy and ligation of esophageal varices to relieve the manifestations of portal hypertension (gastroesophageal bleeding and hypersplenism). There were 51 males and 15 females and their ages varied from 16 to 61 years (mean, 30.4 years). Patients were operated upon at difterent hospitals in the city of Salvador, Bahia, Brazil, but all surgical specimens were examined at the Gonçalo Moniz Research Center. Preoperative diagnoses were made on essentially clinical grounds, but schistosome eggs were demonstrated in the stools from all patients. No information on previous antischistosome chemotherapy could be obtained from the patients. Diagnoses were pathologically confirmed in every case, and portal fibrosis with destruction of the intrahepatic portal vein branches and preservation of arterial and bile ductal structures were the primary, and sometimes the sole, lesion.

\section{Histology}

Fragments of liver tissue were fixed in Bouin's fluid or bulfered $10 \%$ formalin and entuededed in paraffin. Sections were routinely stained with hematoxylin-eosin, Weigert's elastic method, periodic acid-Schiff with and without previous diastase digestion, Picrosirius-red collagen stain, Gomoris reticulum method, Perls' iron stain, and the Shikata's orcein stain for hepatitis virus $B$ surface antigen. Material stained with Picrosirius-red was examined with and without polarized light.

\section{Immunocytochemistry}

The last 18 biopsies were obtained from Roberto Santos Central Hospital in Salvador and followed a different routine. The liver fragments were divided into three parts. One portion was fixed and processed for histologic study as described above. Another portion was embedded in Tissue Tek (Miles Inc, Elkhart. IN) and snap-frozen in liquid nitrogen. Blocks were kept in air-tight bottes at $-70^{\circ} \mathrm{C}$ until the moment they were cut in a cryotome at $-20^{\circ} \mathrm{C}$. The sections were then submited to 
either direct or indirect immunofluorescence techniques for the demonstation of type I, III, pro-HI, IV, and V collagens. at well as for libronectin, laminin, desmin, elastin, and actin. Collagen types l. III. pro-collagen III, and IV were prepared from fibrotic human liver, calf skin, and bovine lens capsule, respectively, after limited pepsin digestion and fractional precipitation with sodium chloride, according to Rhodes and Miller," as modified for human liver by Chevalier et al. " Polyclonal monospecific antibodies against type $V$ collagen were obtained from Institut Pasteur, Paris, France. The purity of the collagen fractions was verified by SDS-polyacrylamide gel electrophoresis. Human plasma fibronectin was prepared by affinity chromatography using gelatin-sepharose 4 b according to Envall and Rouslahti, " purified by DE cellulose chromatography, and serified by SDS-polyacrylamide electrophoresis. Laminin was provided by Dr ( $i$. Martin (National Institutes of Health, Bethesda, MI) ). Antibodies to the antigens listed above were raised in rabhits or goats. Antibodies cross-reacting with common determinants of the different collagen types were diminated by absorption after repeated passages through the different collagens bound to (XNBr-activated sepharose. Other antibodies were commercially obtained: mouse monoclonal anti-alphat actin from smooth muscle (Sigma A 2547, synthetic decapepticlei, potyclonal thicken anti-rabbit actin (Sigma A ghtix, Sigma Chemical Co, St Louis, MO), mouse monoclonal anti-desmin (Amersham RPN 1101 , Arlington, IN), and human polvclonal anti-rahbit elastin ${ }^{12}$ (Institut Pasteur, P'aris. France). Optimal dilution lon the primary antibodies varied from $1: 2$ for anti-iype I collagen to $1: 5$ for all the other antisera, and 1.200 for the monoclomal antibodies (anti-ionin and anti-desmin). The fluoresceinated secondary antibodies were diluted $1: 10$ or $1: 80$. The presence of $S$. mansomi antigen(s) in the inflammatory foci was investigated in eight cases with the use of crotome sections and immunofluorescence microscopy with monse monockonal antibodies against circulating anodic antigen and circulating cathodic antigen, as well as goat polyclonal antisoluble egg antigen.

\section{Electron Microscopy}

The third portion was cut into tiny picces and immediately immersed into iced $0.2 \%$ glutaraldehyde in $0.1 \mathrm{~mm} / 1$. sodium cacodylate buffer, pH 7.4, for 2 hours. Fragnents were washed in the buffer solution, post-fixed for 1 hour in $1 \%$ osmium tetroxide, and embedded in Epon resin. Blocks were cut in a Reichers [ltra cut automatic ultramicrotome (Reichert, Vienna, Austria). Semithin sections were stained with toluidine blue and used for selecting areas for ultrathin sectioning. Ultrathin sections were made with a diamond knife, contrasted with lead nit late and uranyl acetate, and examined with a Zeiss E.M-9 electron micoscope at $50 \mathrm{kV}$.

\section{RESULTS}

\section{General Data}

Portal fibrous enlargement, with partial or total destruction of the main portal vein branch and preservation of the ductal and arterial structures, could be demonstrated in every case. The parenchyma usually maintained its normal lobular or acinar architecture, although dissected by long and thin fibrous septa. Periovular granulomas were not considered essential for the histopathologic diagnosis of hepatosplenic schistosomiasis, but were searched for and registered, their presence being rare, as can be seen in Table 1.
TABLE 1. Main Microscopic Findings in Surgical Liver Biopsies From 63 Patients With Hepatosplenic Manson's Schistosomiasis

\begin{tabular}{lrr}
\multicolumn{1}{c}{ Findings } & No. & \\
\hline Cranulomas & & \\
With eggs & 21 & 31.8 \\
Without eggs & 2 & 3.11 \\
Chronic hepatitis: & 11 & 16.6 \\
Active (mild 5. moderate 6) & 36 & 54.5 \\
Persistent (mild 26, moderate 8, marked 2$)$ & 35 & 53.0 \\
Septal fibrosis & 7 & 10.6 \\
Vodular regeneration & 1 & 1.5 \\
Cholestasis & \\
\hline
\end{tabular}

* Classificatien of chronic hepatitis was made actording to the ariterial of DeGronte el al. ${ }^{13}$

\section{Extracellular Matrix}

Fibrous tissue in portal spaces and in septal bands exhibited variable densities (Fig 1). In zones of dense collagenous tissue one could see areas in which the matrix appeared rarefied with loosely arranged and dispersed fibers in the middle of amorphous cosinophilic ground substance. Sometimes a relatively extensive area of the portal tissue had a fibrillar appearance, with numerous dilated thin-walled blood vessels (angiomatoid (hange) in the absence of inflammatory changes and fibroblast or endothelial cell proliferation. Picrosiriusred staining showed collagen fibers to be fragmented and to exhibil different thicknesses and variable sizes and forms in areas of rarefaction (Fig 2). The same was also detected in sections examined under polarized light.

Fibrous tissue in portal and septal areas showed specific fluorescence for type I collagen. The fluorescence was brighter for type III collagen and, particularly. for pro-collagen III. Usually type I collagen was better seen in areas of more compact fibrosis. Type III collagen had a more diffuse distribution and was the predominant type in septal fibrosis. In areas of portal fibrosis exhibiting focal rarefaction both collagen types could be identified inside and outside the rarefied zones. A quantitative estimation of which collagen type was more abundant seemed impossible with the techniques used. There were variations not only from case to case. but also from area to area. Antibodies against type III and pro-III collagens are probably more reactive and usually yield brighter fluorescence. Type $V$ collagen was seen as thin and dispersed fibers, especially within highly vascularized areas, forming a reticular network. Type IV collagen was scanty within the fibrous tissue but gave good staining for the basement membranes of blood vessels and biliary ducts. The blood vessels were distinctly demonstrated with anti-type IV collagen antibodies and so the angiomatoid lesion of schistosomiasis became more evident. The same happened with anti-laminin. Anti-elastin antibodies not only showed the inner walls of blood vessels, but recognized numerous hyperplastic fibers in the portal spaces and fibrous septa (Fig 3A). The amount of elastic tissue shown by elastic stain (Weigert's) or by immunofluorescence with anti-elastin antibodies was markedly increased. Elastin appeared more concentrated in areas where the portal vein 


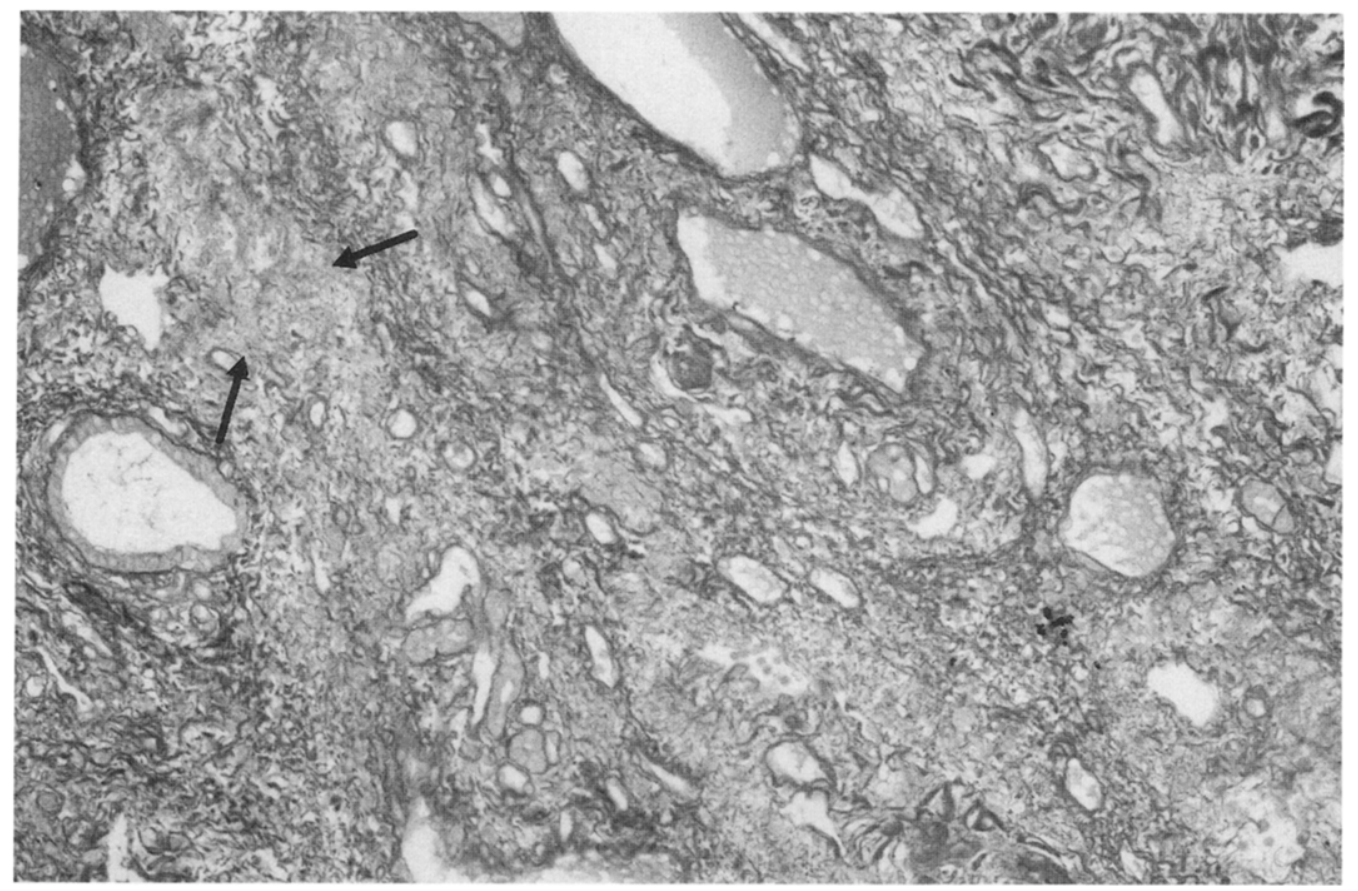

FIGURE 1. A fibrotic and well-vascularized portal space showing focal areas where the collagen tibers have almost disappeared (arrows) and zones of fragmentation of coarse fibers (upper left corner). (Picrosirius-red stain; magnification $\times 90$.)

branches were destroyed, especially around dispersed smooth muscle cells. It was also abundant in subcapsular areas, in zones where the matrix appeared rarefied, and in areas of angiomatoid transformation. Sometimes dark clumps and wavy and fragmented fibers of elastic staining material were observed, suggesting areas of elastic tissue degradation (Fig 4). Fibronectin was abundant in portal spaces, forming a dense network, but sometimes showing areas of rarefaction (Fig 3B). The anti-actin antibodies reacted with the muscular wall of blood vessels and with several structures dispersed within the portal fibrous tissue, identified as dispersed smooth muscle fibers (Fig 3C). Desmin antibodies were a good marker of endothelial cells in the portal vessels (Fig 3D), but at the parenchymal level they stained elongated and plump cells along the sinusoidal walls that probably represented fat-storing cells (Fig 3E).

At the ultrastructural level the matrix usually contained abundant normal-looking, cross-striated collagen fibrils, forming dense and parallel bundles separated by spaces containing long and thin cellular cytoplasmic prolongations or amorphous material, within which a network of fine and straight microfibrils and elastin deposits was seen. Vo clear-cut correlation was obtained

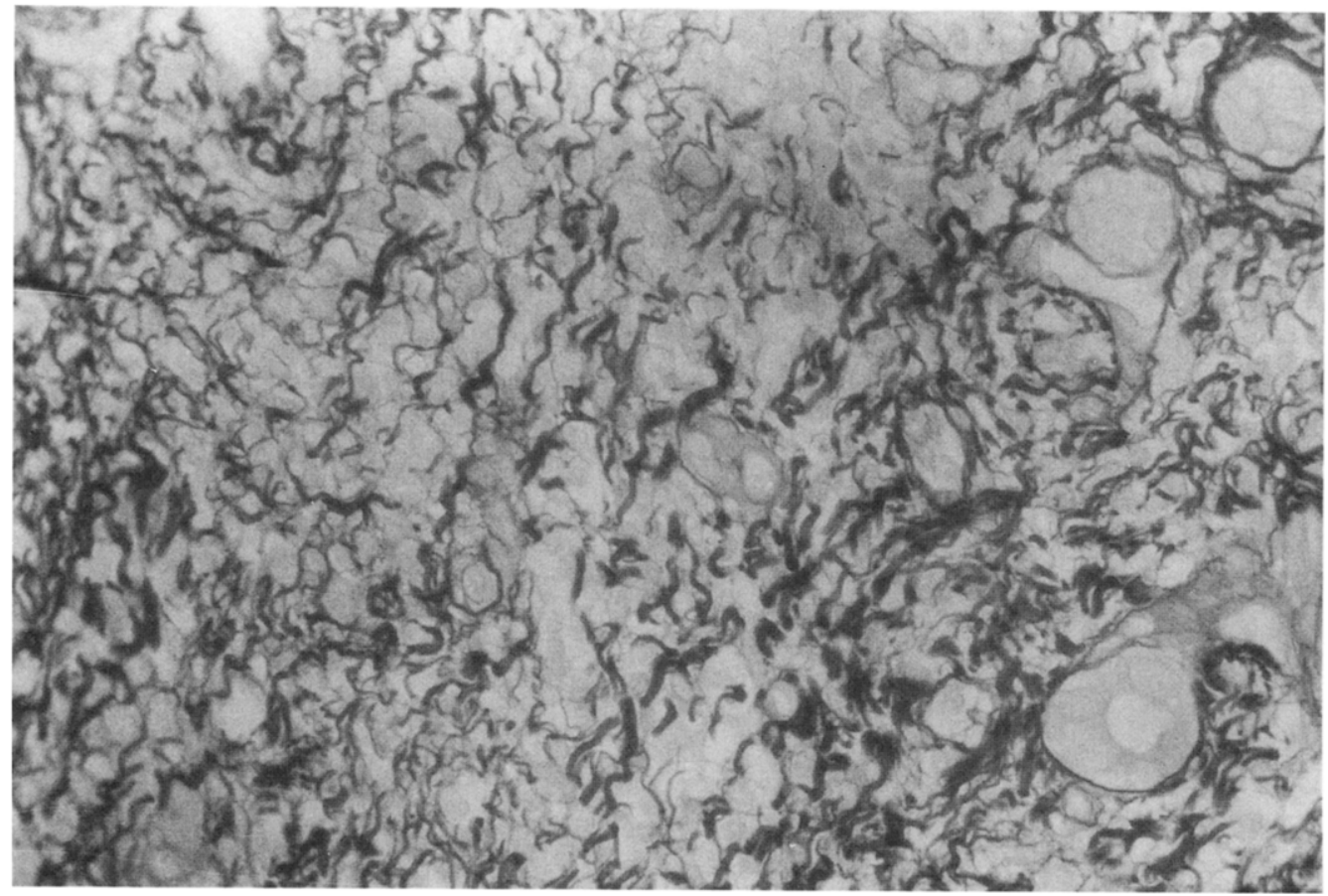

FIGURE 2. Specifically stained collagen fibers present fragmentation, dissociation, and variation in thickness. (Picrosirius-red stain: magnification $\times 356$.) 

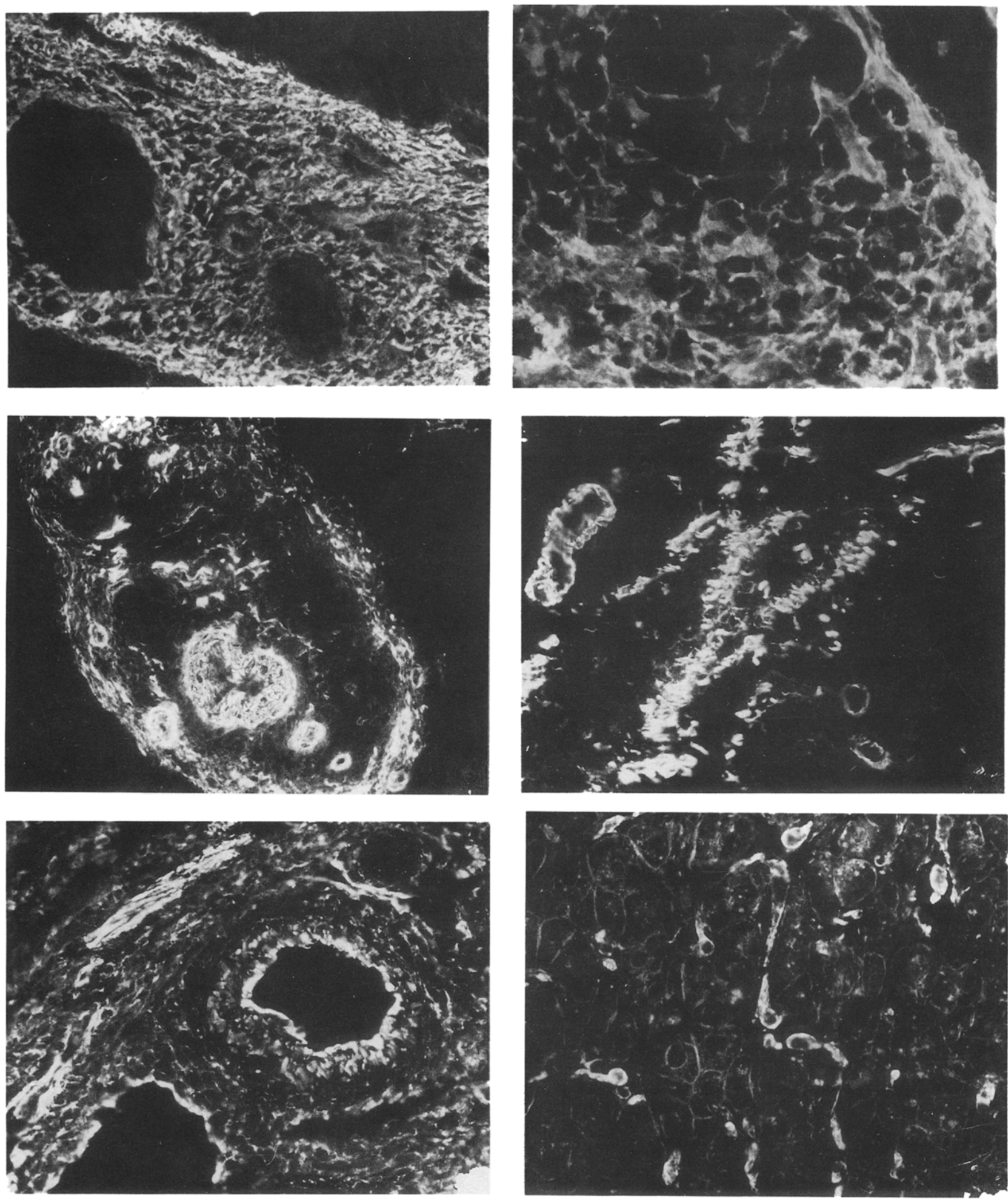

FIGURE 3. (Top left) Presence of increased elastin content in an enlarged fibrotic portal space. (Immunofluorescence: magnification $\times 182$.) (Top right) Fibronectin appears abundantly in schistosomal periportal fibrosis, but with irregular concentration from place to place. The photograph shows a focus of rarefaction. (Immunofluorescence; magnification $\times 364$.) (Center left and right) Portal space stained with specific fluoresceinated antibodies against actin. One can see the wall of blood vessels, but also dispersed fragments of muscular tissue and groups of isolated muscular fibers. (Immunofluorescence: Magnifications: center left, $x$ 182; center right, $\times 364$.) (Bottom left and right) Fluorescent staining for desmin. The endothelial cells in the portal space as well as other connective lissue cells dispersed within the fibrous lissue appear brightly positive. (Immunofluorescence. Magnifications: bottom left, $\times 364$; bottom right, $\times 376$.) (Bottom left) Within the parenchyma the positive cells are elongated and have a parasinusoidal location, probably representing fat-storing cells (Ito cells), while the sinusoidal endothelial cells are negative (bottom right). 


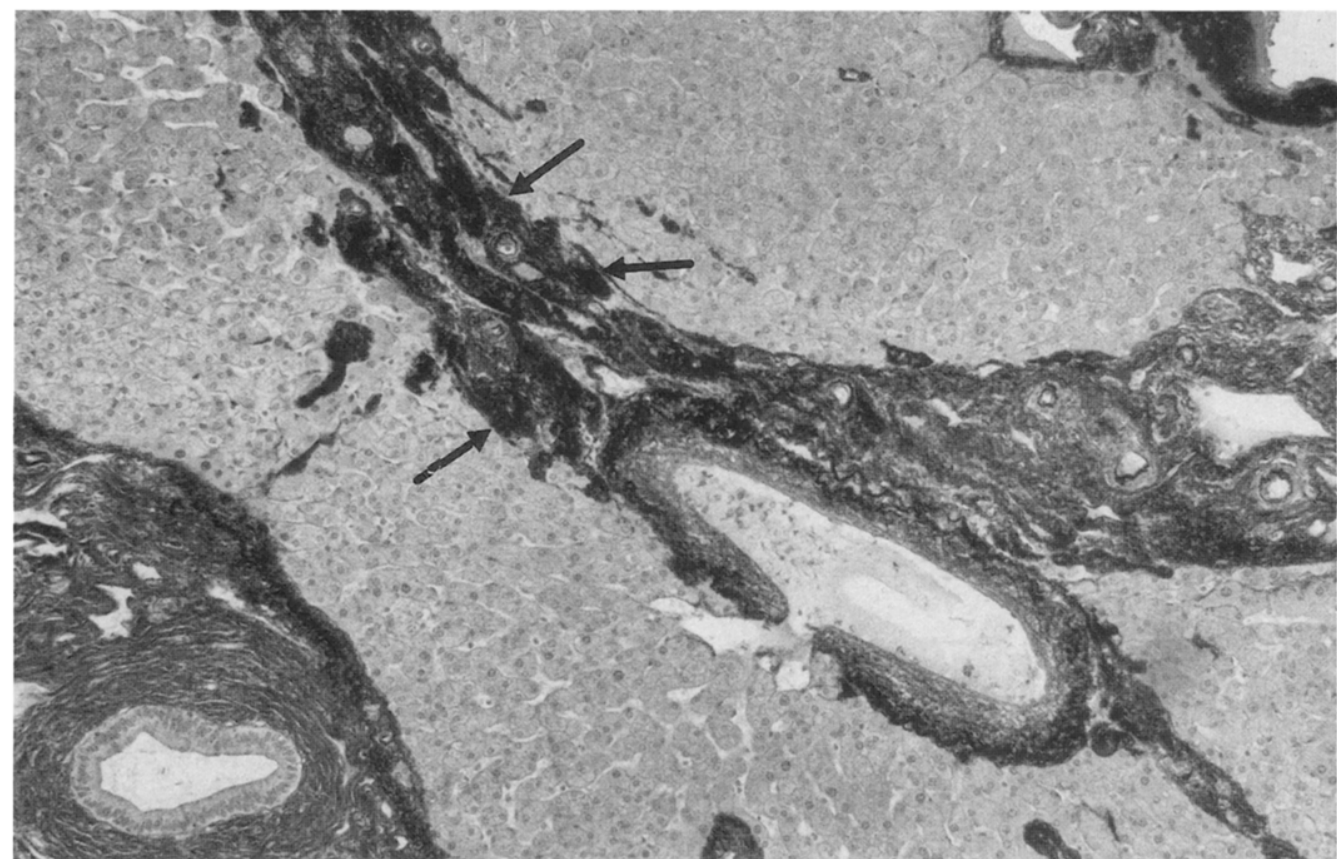

FIGURE 4. Elastic fibers are hyperplastic in the enlarged portal space, sometimes forming dark and structureless clumps (arrows). (Weigert's stain for elastic tissue: magnification $\times 108$.) between these focal arcas of matrix dissolution and the presence of cells, inflammatory or otherwise, although several cellular types could sometimes be identified in the sections (Fig 5). Besides fibroblasts and a few myofibroblasts, eosinophils in different stages of degranulation, neutrophil polymorphonuclear leukocytes, lymphocytes, macrophages, and even mast cells were found in variable numbers within the portal and septal tissues. In the middle of well-differentiated collagen bundles the formation of empty spaces of variable sizes and shapes was often observed. In the vicinity of such spaces collagen fibrils exhibited fragmentation and variations in caliber. In other focal areas of degradation the collagen fibrils were replaced by finely granular electrondense material (Fig 6) with focal collagen fragmentation, dissociation, and the presence of microfibrils.

\section{Vascular Lesions}

Larger portal vein branches frequently showed phleboscleroses, focal or diffuse intimal thickening, thrombosis in different stages of organization, and re-

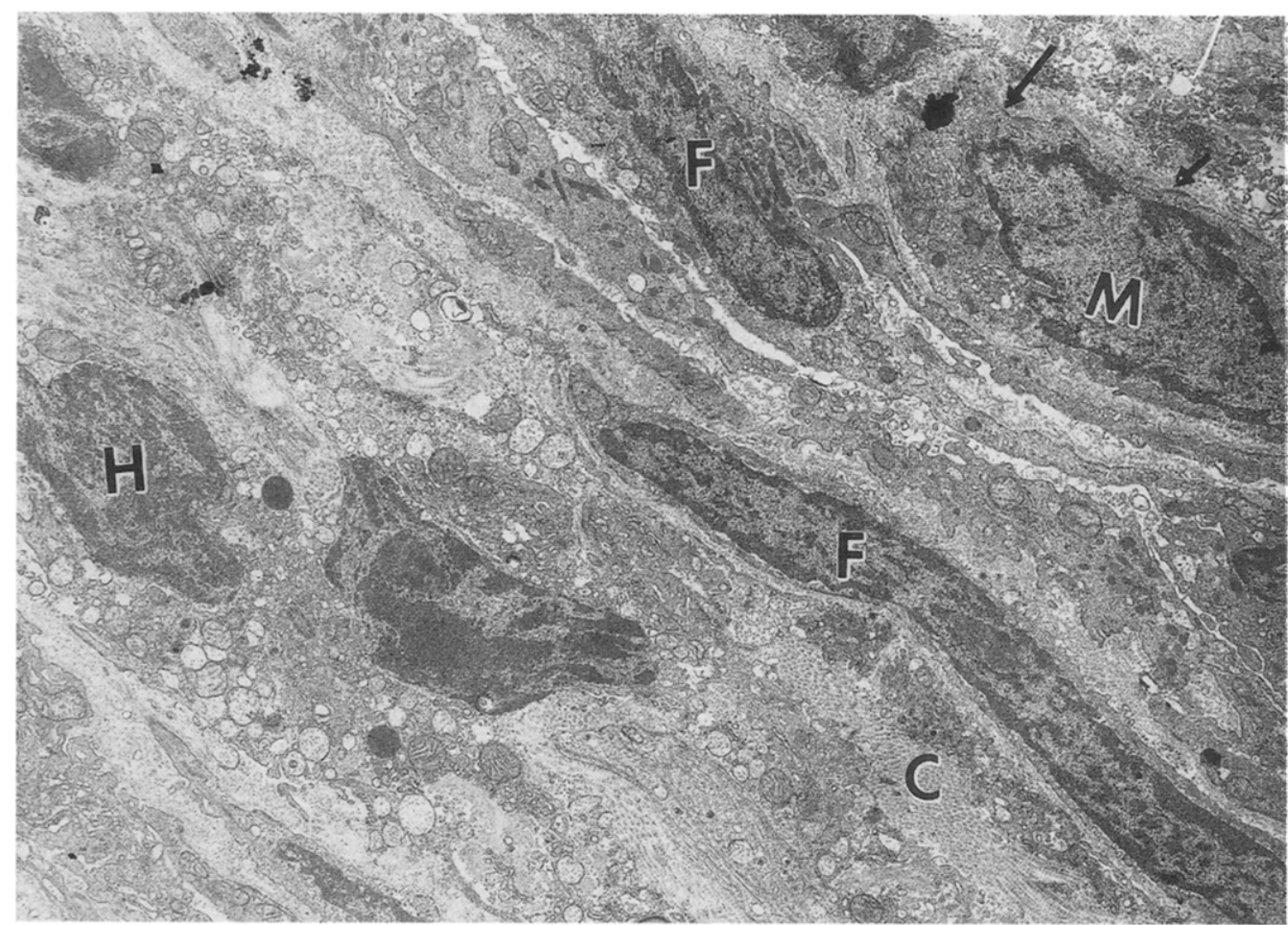

FIGURE 5. Representative area of the portal space in hepatosplenic schistosomiais. There are parallel rows of collagen fibrils (C), separated by fusiform cells, cytoplasmic prolongations, and areas of amorphous substance. $M$, a myofibroblast with a prominent encoplasmic reticulum, a dark, discontinuous submembranous contractil apparatus (arrows), and basement membrane-like material around the cell: $H$, hepatocyte: F, fibroblasts. (Electron micrograph: magnification $\times 2.610$.) 
FIGURE 6. Zones where the collagen fibrils appear interrupted and replaced by dark granular material: "electron-dense changes" (arrows). c. collagen fibrils: cp. cytoplasmic prolongations. (Electron micrograph: magnification * 10,800 .)

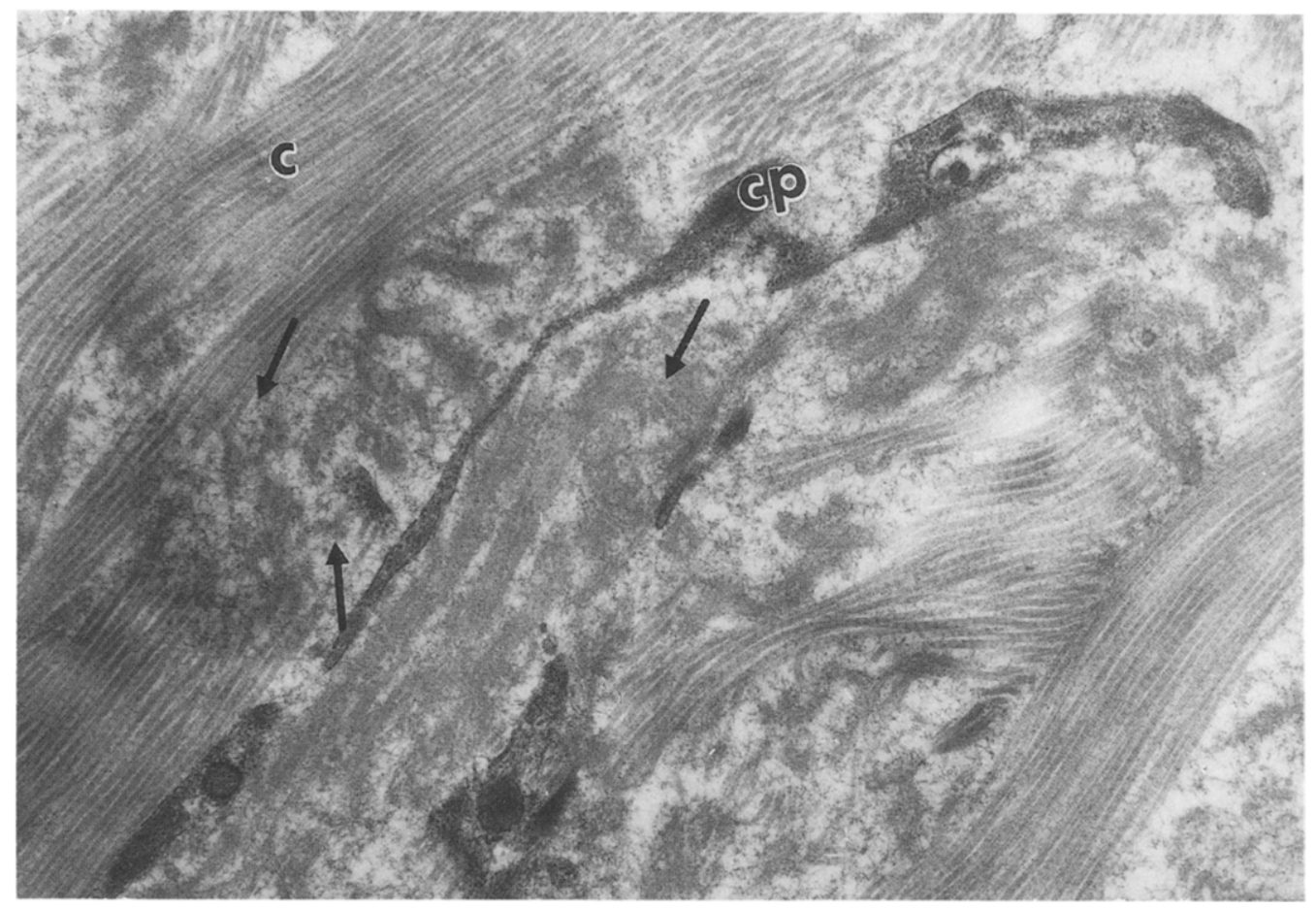

Canalization, natrowing, and retraction. In the presence of such alterations, the muscular wall of the portal vein appeatred disrupted with smooth muscle cells and groups of muscular fibers becoming dispersed in the fibrous portal tissue, a constant finding in the present material (fig 7). The use of fluorescent antibodies against actin helped demomstrate muscular tissue throughout the portal space (Figs 3C and 3D). Schistosomal pigment was frequently noticed in the portal spaces, especially around old periovular granulomas. No increase or decrase in fibrosis. inflammation, or any other feature was found in relation an the presence of pigment.

\section{Chronic Hepatitis}

The presence of inflammanory changes in the portal spaces, other than those around the schistosomse egos, was detected in several cases. One case disclosed a positive Shikata lest. This case presented chronic active hepatitis and partial nodular iransformation of the hepalic parenchyma. All the other adses were negative.

As for inflammatory changes, there were focal accumulations of macrophages and lymphorytes in the middle of the langer portal areas, hut not in the smaller porlal lracts. The presence of eosinophils varied from

FIGURE 7. Portal changes in advanced schistosomiasis. The portal space is enlarged and fibrosed, with preservation of the arterial (a) and ductal (d) structures, but with partial destruction of the main portal vein branch $(v)$, which shows phlebosclerosis and dispersion of the muscular fibers (arrows). Many thin-walled dilated blood vessels also appear throughout the portal area (angiomatoid change). (Hematoxylin-eosin stain; magnification $\times 210$.

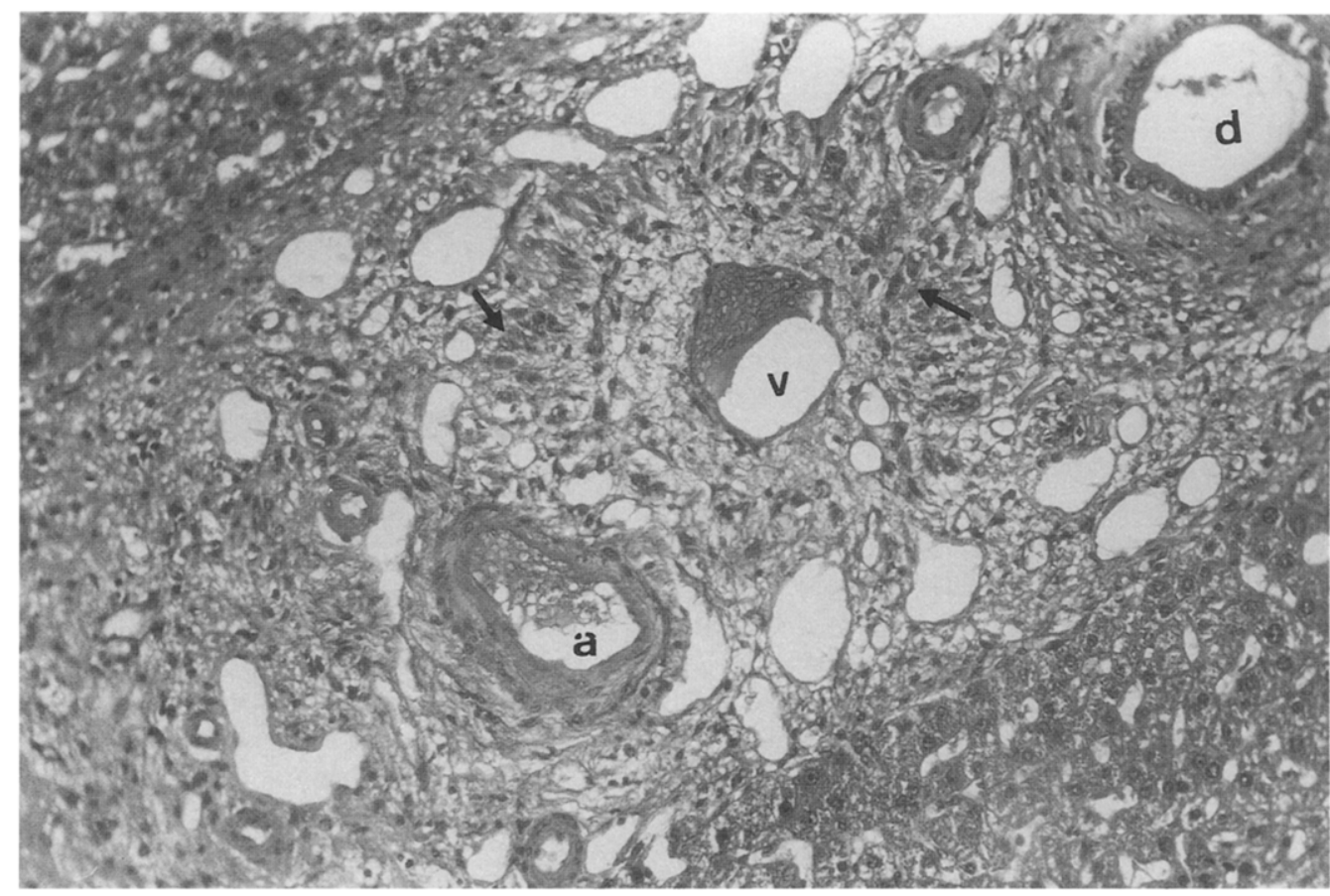


moderate to mild. Accumulation of mononuclear inflammatory cells also occurred between the parenchymal border and the portal fibrous tissue, especially in the larger portal spaces, but still maintained a focal pattern (Fig 8). Infiltration and dissociation of the liver cell cords at the parenchymal border ("piecemeal" necrosis) were frequently observed. No correlation could be established between the presence of nonspecific hepatitis and the periovular granulomas

The presence of schistosome antigen(s) was tested in eight cases. Five cases exhibited hepatitis and three did not. None had schistosome eggs in the sections examined. Results were essentially negative in all cases.

\section{DISCUSSION}

The diagnosis of advanced hepatic schistosomiasis in surgical biopsy material does not constitute a great problem. The presence of portal enlargement due to fibrosis, the partial or lotal destruction of portal vein branches, the preservation of ductal and arterial structurcs, and the maintenance of parcnchymal lobular or acinar architecture are usually sufficient for a positive diagnosis, since these combined changes are usually not to be found in other conditions. However, when examining material from endemic areas, sometimes one sees focal fibrosis with vascular proliferation and ectasia (angiomatoid formation), especially in the presence of cirrhosis. In such cases the diflerential diagnosis with schistosomiasis can be crucial. The search for muscular cells or fibers "buried" in the fibrous tissue will frequently offer a key for the diagnosis of schistosomiasis. This finding is an indication that a small or mediumsized branch of the portal vein has been destroyed, an indispensable feature to characterize the periportal fibrosis as being of schistosomal origin. The presence of isolated smooth muscle fibers within the portal tissue was observed in every case of the present series. Although immunofluorescence for actin can make this finding more impressive, light microscopy with conventional staining is entirely adequate. Some degree of phleboscleroses with dissociation of the portal vein muscular coat may appear in cirrhosis and in other fibrosing hepatic processes, but cannot be compared with the profound disarray usually present in advanced schistosomiasis.

Dislodged muscle cells in portal tissue remain viable. Therefore, they may be important for the contractility of the fibrous tissue and may play a role in the pathogenesis of portal hypertension. They may differentiate into myofibroblasts, which have been recognized as an important cell type in periportal schistosomal fibrosis. ${ }^{14}$ Studies in vitro have shown that connective tissue cells isolated from human schistosomal fibrosis exhibited a modified phenotype of smooth muscle cell. ${ }^{15}$ Smooth muscle cells also may be engaged in the synthesis of elastin, ${ }^{16}$ another matrix component abundant in pipestem fibrosis. Morphologically, there was good correlation between the presence of elastin on elastic fibers with signs of portal vein destruction and muscular dissociation. Endothelial cells may also synthesize elastin. ${ }^{16}$

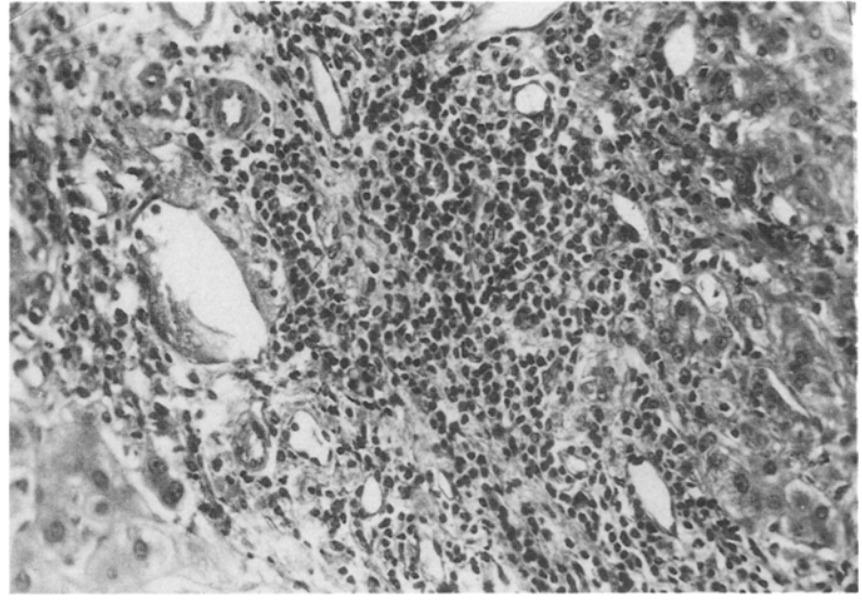

FIGURE 8. Chronic hepatitis with a mild degree of activity in schistosomiasis. Small portal spaces show infiltration by inflammatory mononuclear cells with mild extension into the parenchymal border, a picture representative of the type of chronic hepatitis associated with advanced Schistosomiasis mansoni. (Hematoxylin-eosin stain; magnification $\times 164$.)

The presence of numerous endothelial cells in the angiomatoid lesion and the dislodgement and proliferation of smooth muscle cells may explain the prominent elastic hyperplasia in advanced hepatic schistosomiasis. However, elastic fibers are conspicuously absent from periovular granulomas. ${ }^{17.18}$ It has been suggested that elastic tissue in liver disease is related to chronicity of the lesions. ${ }^{19}$ Perhaps an isolated periovular granuloma may not survive the necessary time for excess elastin synthesis to take place.

Matricial changes in schistosomiasis are complex and dynamic. There are different genetic types of collagens, associated proteins, and abundant proteoglycans. $^{\text {is }}$ Collagen fibers show focal ultrastructural changes compatible with chronic degradation of the same morphologic types as those found in experimental periovular granuloma after chemotherapy. ${ }^{201}$ Most of the patients in the present series could not recall being treated for schistosomiasis, which means that degradation of collagen may occur spontaneously after partial or total self-cure of the infection. Spontaneous regression of the hepatosplenic form of schistosomiasis has been recorded," ${ }^{21}$ as well as involution following chemotherapy. ${ }^{22.23}$ We observed that morphologic evidence of collagen degradation can be detected by light microscopy. It is the counterpart for the involution of periportal fibrosis that can now be followed by ultrasonography in treated patients. ${ }^{23.2-4}$ Excess fibrous tissue in portal spaces is far from having a homogeneous appearance. It shows areas of rarefaction and of actual fragmentation of fibers. These changes are not always properly evaluated by the pathologist. It is expected that the whole subject of periportal schistosomal involution now being described in humans may stimulate a closer microscopic observation of collagen changes within the concept of chonic matrix degradation. ${ }^{20}$

Actually, fibrosis results when synthesis of extracellular matrix exceeds degradation, both processes oc- 
"urring side by side. The natural tendency is toward a balanced parenchyma/stroma ratio. ${ }^{26}$ Today, the use of large-scale chemotherapy for schistosomiasis and portable ultrasound technology has permitted the documentation of slow resorption of portal fibrosis. In a certain proportion of cases, degradation of the fibrous matrix is probably followed by remodeling of the obstructive, intrahepatic vascular changes, which are primarily responsible for portal hypertension, with complete reversion of hepatosplenic disease. ${ }^{2-2}$ However, the rendency of the fibrous tissue to undergo resorption probably occurs in every case in which the schistosomal infection has become inactivated. Matrix degradation is probably more vigorous in carly treated infections, but no data are available to substantiate this claim. Our palients did not provide a history of previous treatment. The cases of posttherapeutic periportal fibrosis involution so far reported have not included pathologic data.

The changes of chronic hepatitis seen in hepatosplenic schistosomiasis remain obscure in their pathogenesis and significance. When there are chronic active hepatit is and progression to cirrhosis, an associated infection with the $B$ hepatitis virus can usually be demonstrated." ${ }^{2 i}$ However, such an association is difficult to prove in those more frequent cases of chronic persistent or mildly active hepatitis. These latter cases had some peculiarities. such as the presence of focal accumulation of mononuclear cells in the middle of the fibrotic area and the presence of invasion of the parenchymal border by inflammatory cells in larger rather than smaller portal spaces. These aspects seem related to the schistosome infection, since no other cause was clinically or morphologically apparent in the material studied. However, it was not possible to demonstrate schistosomal antigen(s) in relation to these inflammatory changes. Techniques of immunoelectron microscopy may be necessary in future sudies to help clarify this point.

Acknowledgmont. Thanks are due to Dr A. M. Deelder, l.eiden, Holland, for providing the antischistosoma sera and (1) Dr Allen W. Cheever, National Institutes of Health, Bethesdit, MI), for reviewing the manuscript.

\section{REFERENCES}

1. World lfeath (Mganization: (ontrol de la esquistosomiasis. sories de Informes lécnicos 798: 1-126, 1985

2. Che ever AW': A quantitative post mortem study of Schistosomiasis mansmi in mat1. An f Trop Med Hyg 17:38-64, 1968

3. Colley DC, Gircia AA, I ambertuci JR al: Immune responses during human shistosomiasis. XIl. Differential responsiveness in patients with hepatosplenic disease. An J Trop Med Hig 35:793$80 \% .1986$

4. [weards (; Osman (iS, E] Koly A. et al: Failure of immunosuppressive nechanisms in human Schistesoma mansoni infection with hepato-splenomengats. J C.Iin Microbiol 25:768-773. 19187
5. Symmers W'Sic: Note on anew form of line cirrhosis due 10 the presence of the ona of Bitharzia hatmatodia. I Pathol Bacteriol !l: $2.37-239,1904$

6. Andrade ZA: Evolution and involution of hepatesplenic schistomomiasis. Mem Inst Oswaldo (Cruz $84: 58-75,1989$ (suppl 1)

7. MeCully RM, Barrone C:N, Cheever AW: Sohiseosomiasis (Bilharsiasis). in Binford ( $: H$. Comnor DH (eds): Patholeng of Tropical and Fxtraordinary Diseases, vol 2 , section 10 , chapper 1. W'ashington, 1) A. Amed forces Institute of Pathologs. 19/h, pp 182-5018

8 . Bogliolo $\mathrm{L}$ : The anatonical picture of the liver in hepatosplenic Schivesomiasis mansomi. Ann Trop Med Parasitol 51:1-14, 1957

9. Rhodes PK, Miller F.J: Physical characteriration and molectulat organization of the collagen $A$ and $B$, hains. Bich hemistry 17:34.42$3.48,1978$

10. Chevalier (), Herbage D), (irimatud fA: Collagen polymorphism in human liver in Biochemistry of Vomal and Pathological Connective Tissue. bo. Colloquium of the Ferteration of Ruropean Connective Tissue Clubs, wol I. Centre National de la Recherche Scientifique. Creteil, $1978, p g \times 2$

11. Fingrall F, Ruostahti $k$ : Binding of soluble fomm of tibroblats surface protein, fibronectin to collagen. Inst J Cancer 20:1-5, 1977

12. Lethias C, Hartman DJ, Masmejean M, et al: IItrastructural immunolocalization of elistic fibers in rat bloced ve'ssels using the prorein A-gold technique. J Histochem Cyochem 35:15-91, 1987

13. DeCroote J. Desmet VJ. Gedigk P, et al: A classification of chronic hepatitis. Iance $2: 626-628,1968$

14. Crimaud JA, Borojevic R: Mvofibroblasts in hepatic schistosomal fibrosis. Fxperientia 33:890-892, 1977

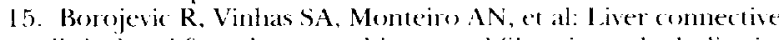
tissue cells isolated from human schisosomal fibrosis or alcoholic cirrhosis represent at modified phenotype of smooth muscle ell. Biol (cell $53: 231-238,1985$

16. Davidson JM: Elastin. Structure and biology in Llitu J, Pareja A| (eds): Comnective Tissue Disease. Moleculat Pathology of the (ionnective Tissue, New York, NY, Dekker, 1987, pp 299-5.3

17. Andrade ZA, Grimaud JA: Evolution of the schistosomal hepatic lesions in mice after curative chemotheraps. Am J Panol 1'4 $54-65,1986$

18. Junqueira I.CU, Montes (BE, Toledo oMS, et al: Morphological, histochemical and biochemical observations on the connective tissue natrix of in sith and isolated hepatic granulomas in experimental murine schisosomiasis. Ann Trop Med Parasitol 80:27-41, 1986

19. Liban F. Hungar MD: Elastosis in fibrotic and cirrhotic processes of the liver. Arch Pathol 68:331-341, 1959

20. Andrade ZA, Grimaud JA: Morphology of chronic collagen resorption. A study on the late stages of sh histowmal granuloma involution. Am J Pathol 132:389-399, 1988

21. Kali N. Brener $Z$ : Evoluca ón clinica de 112 asos de esquistossomose mansoni observados após 10 anos de permanencia em focos condêmicos de Minas (Ferais. Rev Ins1 Med Trop San Paulo 8:1391-1 42. 1966

22. Bina JC.. Prata A: Regressão da hepatenplenomegalia pelo tratamento especifico da esquistossomose. Rev Soc Bras Med Trop $16: 2] 3-218,198: 3$

23. Honeida MA, Fenwick A, DeFalla AA, it at Effert of antishistosomal themotherapy on prevalence of Symmers' periportal tibrosis in Sudanese villages. Iancet 2:437-439, 1988

24. Coutinho $\mathrm{AD}: \mathrm{A}$ new dynamic approach to the diagnosis of Symmers' fibrosis in schistosomiasis by ultrasound. Rev lnst Med 'Trop Sao Paulo 32:73-77, 1990 (editorial)

25. Perez-Tamayo R: Collagen resorption in carrageenin granuloma. II. Llt rast ructure of collagen resorption. Lah Invest 22:142159.1970

26. Perez-Tamayo R: Some aspects of comnective tissue of the liver in Popper H. Schatfner F (eds): Progress in Liver Diseases, vol 2. Philadelphia, PA, Grune \& Stratton, 1965, pp $194-210$

27. Lyra LC, Rebrousas (;, Andrade ZA: Hepatitis B surface antigen carrier state in hepatosplenic schistosomiasis. Gastroenterology $71: 641-645,1976$ 